\title{
Flap Selection Algorithm Based on the Body Mass Index for Phalloplasty in Female-to-Male Transgender: Techniques and Outcomes
}

\author{
Toshiyuki Watanabe, $\mathrm{MD}, \mathrm{PhD}^{1}$ Yuzaburo Namba, $\mathrm{MD}, \mathrm{PhD}^{1}$ Yoshihiro Kimata, MD, $\mathrm{PhD}^{1}$ \\ ${ }^{1}$ Department of Plastic and Reconstructive Surgery, Okayama University \\ Hospital, Plastic and Reconstructive Surgery, Okayama, Japan \\ J Reconstr Microsurg Open 2021;6:e57-e62. \\ Address for correspondence Toshiyuki Watanabe, MD, PhD, Okayama \\ University Hospital, Plastic and Reconstructive Surgery, 2-5-1, \\ Shikata-cho, Okayama, 700-8558, Japan \\ (e-mail: watanabetoshiii@yahoo.co.jp).
}

\begin{abstract}
Background Various phalloplasty methods have been reported; however, a consensus has not been reached. Therefore, we suggest an alternative algorithm for an ideal phalloplasty considering the factors that affect flap selection.

Methods We retrospectively examined 32 phalloplasty cases of female-to-male transgender. Flaps used for phalloplasty were classified into three groups as follows: (1) radial forearm (RF) group, (2) anterolateral thigh (ALT) group, and (3) flap combination (FC) group. Statistical analyses were performed to determine the significance of age and body mass index (BMI) as well as differences in the rates of postoperative complication, reoperation, esthetic acceptability, tactile or erogenous sensibility, and the ability to urinate while standing.

Results Ten cases were included in the RF group and 7 and 15 cases in the ALT and FC groups, respectively. With respect to BMI, the RF group (mean [standard deviation $(S D)]=25.9$ [3.3]) showed significantly higher BMI than the ALT (21.7 [0.9]) or FC group (22.2 [4.5]). The RF (mean $[S D]=3.7[0.4]$ ) and ALT groups (3.5 [0.9]) had significantly higher esthetic acceptability evaluation scores (from 1 to 4 ) than the FC

\section{Keywords}

- body mass index

- phalloplasty

- female-to-male transgender group (2.5 [1.2]). Moreover, the RF and ALT groups were superior than the FC group in terms of tactile or erogenous sensibility rates.

Conclusion BMI effected flap choices for phalloplasty because of the correlation between BMI and skin thickness. FC becomes an option between ALT flap and RF flap in terms of flap choice based on BMI. Our alternative algorithm for an ideal phalloplasty based on BMI will be useful for objective flap choices.
\end{abstract}

Phalloplasty was first described by Bogoras in 1936, and it consisted of a pedicled abdominal tubed flap implanted with rib cartilage. ${ }^{1}$ In 1946, Maltz reported the concept of phalloplasty with a "tube-within-a-tube" flap design, and it became widely used. ${ }^{2}$ Since then, the use of various pedicled flaps, such as the abdominal, scrotal, tubular thigh, and gracilis flaps,

received

November 2, 2020 accepted after revision May 7, 2021
DOI https://doi.org/ 10.1055/s-0041-1732373. ISSN 2377-0813. has been reported. ${ }^{3-6}$ However, limitations have also been reported for each technique, including the necessity of multiple surgeries, unsatisfactory sensory recovery, impaired flap circulation, and numerous scars at the donor site. ${ }^{3-6}$

Chang and Hwang first reported phalloplasty using a free flap in $1984 .^{7}$ The free radial forearm (RF) flap is thin and

This is an open access article published by Thieme under the terms of the Creative Commons Attribution-NonDerivative-NonCommercial-License, permitting copying and reproduction so long as the original work is given appropriate credit. Contents may not be used for commercial purposes, or adapted, remixed, transformed or built upon. (https://creativecommons.org/ licenses/by-nc-nd/4.0/)

Thieme Medical Publishers, Inc., 333 Seventh Avenue, 18th Floor, New York, NY 10001, USA 
soft, easily accommodates the sensory nerves, and has produced satisfactory esthetic and functional results. Since then, various types of flap methods have been reported, such as a dorsal pedis flap, deltoid flap, cricket bat flap, lateral arm flap, fibula flap, pedicled iliac flap, an anterolateral thigh (ALT) flap, and flap combination (FC) phalloplasty. ${ }^{8-15}$

The requirements for an ideal phalloplasty according to Gilbert et $\mathrm{al}^{8}$ include the following: (1) a one-stage surgery, (2) the ability to urinate while standing, (3) tactile or erogenous sensibility, (4) enough bulk to tolerate the insertion of a penile prosthesis, (5) esthetic acceptability, (6) a small and inconspicuous scar at the flap donor site, and (7) no functional loss at the flap donor site. Based on these criteria, from a functional and esthetic perspective, phalloplasty using an RF flap may provide the best outcome and has often been reported as the gold standard. ${ }^{16}$ Recently, the ALT flap has been reported as the second best choice, and its benefits include the following: no need for expertise in microsurgery, no visible scars, slight functional loss at the flap donor site, and no sacrifice of major blood vessels. ${ }^{14,17-19}$

However, few reports have examined the indicators for flap selection for an ideal phalloplasty. We present our experience by retrospectively studying 32 female-to-male transgenders (FTMTs) and examining the factors that affected flap selection. Furthermore, based on our results, we suggest an algorithm for flap selection in phalloplasty.

\section{Methods}

In Japan, gender identity disorders have been diagnosed and treated in accordance with their guidelines since 1996. Various experts, including psychiatrists, urologists, gynecologists, plastic surgeons, and psychotherapists, joined to establish standard diagnosis and treatment policies. ${ }^{20}$ This study was approved by the ethics committee of the hospital (study no. 2006-041). The participants were 32 FTMTs who underwent flap phalloplasty under general anesthesia at the gender center of the hospital between January 2001 and June 2019. Informed consent was obtained from all study participants. The flaps used in the phalloplasties were classified into the following groups: RF group (RF flap phalloplasty using a "tube-within-a-tube" flap design), ALT group (ALT flap phalloplasty using a "tube-within-a-tube" flap design), and FC group (multiple flaps collected and combined for phalloplasty). $2,7,14,15,17$

\section{Surgical Technique}

Prior to phalloplasty, urethral distraction was performed with a perineal flap, vaginal flap, or metoidioplasty. The absence of fistula in the extended urethra was confirmed by urethroscopy and urethrography. Hormone therapy was discontinued 4 weeks before surgery, and smoking was also interrupted 4 weeks before surgery and was not allowed until 4 weeks postoperatively. Laser hair removal in the planned flaps was performed prior to surgery. The thickness of the flap collection site desired by the patient was measured by ultrasonic images, and a flap with a thickness of 4 to $7 \mathrm{~mm}$ from the epidermis to the fascia was selected.
RF flap phalloplasty used a "tube-within-a-tube" flap design., ${ }^{2,7}$ The radial artery and concomitant veins were anastomosed to the lateral circumflex femoral artery and the accompanying veins in the RF flap. Two cutaneous nerves of the RF flap were anastomosed with two dorsal clitoral nerves.

Pedicled ALT flap phalloplasty also used a "tube-within-atube" flap design. Multidetector row computed tomography angiography (MDCTA) was preoperatively performed to confirm the location of two or more perforators that would be included in the ALT flap. If dominant perforator vessels were not identified, the contralateral ALT flap, RF flap, or FC was alternatively selected. Two lateral femoral cutaneous nerves of the ALT flap were anastomosed with two dorsal clitoral nerves.

In FC phalloplasty, multiple flaps are used: the urethral side is constructed with an RF flap, and the penile shaft is covered with a deep inferior epigastric artery perforator (DIEP) flap, a superficial circumflex iliac artery perforator (SCIP) flap, or an ALT flap. ${ }^{15}$ Other combination patterns include bilateral SCIP flaps, a DIEP and an ALT flaps, SCIP and ALT flaps, an RF flap with SCIP and ALT flaps, and bilateral DIEP flaps. Because containing the cutaneous nerves in the flap when using DIEP and SCIP flaps is challenging, the clitoris is fixed at the base of the reconstructed penis. In this study, there were no specific criteria for flap selection. The flap selection was decided following a discussion between the surgeon and the patient. In phalloplasty with RF or ALT flaps, preoperative echo images confirmed that the flap thickness was 4 to $6 \mathrm{~mm}$, so that intraoperative thinning of the flaps was not required. In FC, thinning was not performed on RF flaps but the thickness of the flaps to be combined was adjusted by thinning the ALT, SCIP, or DIEP flaps.

Postoperatively, the patients were placed on bed rest for 7 days. Anticoagulants (12,000 units of heparin sodium per day for 7 days) were administered to patients with postoperative complications related to microsurgery. These included cases of vascular reanastomosis or patients with a history of thrombosis. All patients were hospitalized for 2 weeks. The urinary catheter was removed 3 weeks after the surgery. The patients were instructed to self-dilate the reconstructed penile urethra to elongate the vaginal urethra once a day for 6 months postoperatively using a 14-Fr catheter.

All surgeries were performed in a single stage. The rate of early postoperative complications occurring within 3 weeks and late complications occurring 3 weeks postoperatively were examined. Early postoperative complications included thrombosis of vascular anastomosis and flap necrosis, and late complications were fistula formation and urethral calculus. Furthermore, the number and reasons for additional surgeries performed were registered.

At least 6 months after surgery, the success rate of phalloplasty was evaluated using the following three criteria: (1) esthetic acceptability, (2) tactile or erogenous sensibility of the phallus, and (3) ability to urinate while standing. Esthetic acceptability was determined by two plastic surgeons specializing in genital medicine using a four-step rating scale (1: strongly disagree, 2 : disagree, 3 : agree, and 
4: strongly agree). This rating scale for esthetic acceptability considered the length, thickness, shape, and adequacy of the reconstructed penis for the patient's body size. For phallus sensory evaluation, the patient was asked regarding the existence or absence of tactile or erogenous sensibility. The occurrence of urethral strictures and fistulas was examined by urethrography and urethroscopy 6 months postoperatively, and the ability to urinate while standing was also documented.

Statistical analyses were performed using the Bell Curve for Excel version 2.0 (Social Survey Research Information, Tokyo, Japan). The Kruskal-Wallis test was performed to determine the factors affecting flap selection in all three phalloplasty groups. The factors considered for this analysis were age, body mass index (BMI), thrombosis rate, flap necrosis rate, fistula rate, calculus rate, fistulectomy rate, open resection depilation rate, esthetic acceptability evaluation score, tactile erogenous sensibility, and the ability to urinate while standing. In addition, for each factor showing significant differences between groups using the KruskalWallis test, the Mann-Whitney $U$-test was used to confirm the significance of the observed difference. A p-value of 0.05 was considered significant.

\section{Results}

The types of reconstruction techniques performed consisted of RF flaps, ALT flaps, and FC in 10, 7, and 15 patients, respectively. Representative cases of each group are shown in - Fig. 1. In two cases, RF flap was changed to ALT flap due to the negative Allen test results.

No significant differences between groups were found in the rates of early and late postoperative complications or in the rate of additional surgeries. In contrast, significant differences were found in BMI, esthetic acceptability, and tactile or erogenous sensibility ( $p<0.05 ;-$ Table 1$)$. In addition, these variables were compared between the RF and ALT groups, RF and FC groups, and ALT and FC groups using the MannWhitney $U$-test. Significant differences were observed for BMI between the RF and FC groups $(p<0.05)$ and between the RF and ALT groups $(p<0.05)$.

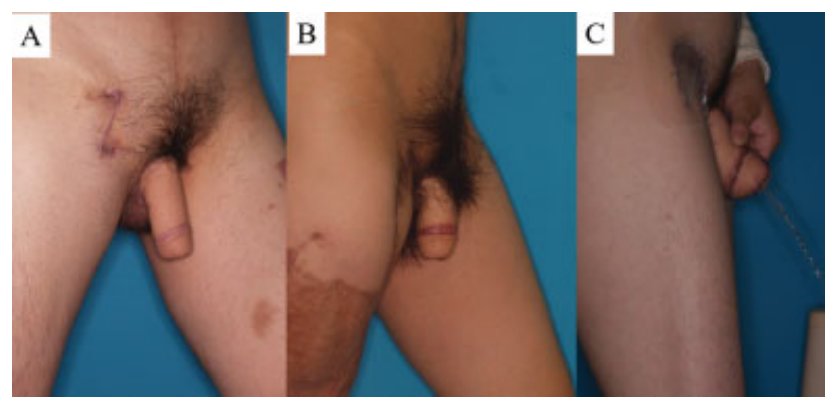

Fig. 1 Postoperative course. Each patient's condition 6 months postoperatively. (A) RF flap phalloplasty case using a "tube-within-atube" flap design. (B) Pedicled ALT flap phalloplasty case using a "tube-within-a-tube" flap design. (C) FC phalloplasty case. The urethral side is constructed with an RF flap, and the penile shaft is covered with a DIEP flap. ALT, anterolateral thigh; DIEP, deep inferior epigastric artery; FC, flap combination; RF, radial forearm.
The RF group had a higher BMI value than the other two groups (-Fig. 2). With respect to esthetic acceptability, the FC group had the lowest score among the three groups, and a significant difference was observed between the RF and FC groups ( $p<0.05$; -Fig. 3 ). Tactile or erogenous sensibility was significantly different between the RF and FC groups and between the ALT and FC groups. Tactile or erogenous sensibility was reported to be lower in the FC group $(p<0.05)$.

\section{Discussion}

Consensus on flap selection for an ideal phalloplasty has not yet been reached because of the complexity of the procedures involved and inconsistency of the postoperative results. ${ }^{18,19}$ While there are several reports on the postoperative complication rates after phalloplasty with RF flaps, there are a few reports regarding the complication rates after phalloplasty with ALT flaps or FC. In a systematic review of 11 papers on phalloplasty with RF flaps, the overall postoperative complication rate was 0.88 per patient. Among the postoperative complications, the stricture or fistula formation rate was 0.2 to 0.8 per patient ( mean $=0.51$ ); 9 to $100 \%$ (mean $=81.7 \%$ ) patients achieved acquisition of tactile or erogenous sensibility and 40 to $99 \%$ (mean $=75 \%$ ) achieved the ability to urinate while standing. ${ }^{19,21}$

In our 32-case series, the overall postoperative complication rate was 0.68 per patient; when comparing the three reconstruction techniques, no significant differences were recognized. Among the postoperative complications, the fistula and stenosis formation rates were 0.59 per patient. The tactile or erogenous sensibility acquisition rate was $75 \%$. The procedures achieved a standing micturition rate of 84\% (-Table 1). Our postoperative results corroborate the findings of the previously mentioned systematic review of 11 papers on phalloplasty with RF flaps. ${ }^{19}$

Significant differences were found in BMI, esthetic acceptability, and tactile or erogenous sensibility between groups. BMI is one of the crucial factors affecting flap selection. In women, a higher BMI has been reported to increase subcutaneous fat around the thighs and abdomen. ${ }^{22,23}$ To develop an acceptable shape for the penis, the flap thickness should be approximately 4 to $6 \mathrm{~mm}$. In the tube within tube style phalloplasty design, the urethral circumference into which the urethral catheter $14 \mathrm{Fr}$ can be inserted is approximately $37 \mathrm{~mm}$. When tube within tube style phalloplasty is performed based on the urethral circumference of $37 \mathrm{~mm}$, if the flap thickness is $4 \mathrm{~mm}$, the flap collection width is $152 \mathrm{~mm}$. Similarly, if the flap thickness is $5 \mathrm{~mm}$, the flap width is $167 \mathrm{~mm}$; if the thickness is $6 \mathrm{~mm}$, the flap width is $181 \mathrm{~mm}$; and if the flap thickness is $7 \mathrm{~mm}$, the flap width is $196 \mathrm{~mm}$. With the flap thickness increased by $1 \mathrm{~mm}$, the tube within tube style phalloplasty requires an extension of the flap width of approximately $1.5 \mathrm{~cm}$.

In our series, the circumference of the forearm is approximately $18 \mathrm{~cm}$ near the wrist joint, and when using RF, if the thickness is $8 \mathrm{~mm}$ or more, we need to use two flaps or perform additional skin grafting on the flap. With ALT flaps, if the ALT flap is collected with a width of $196 \mathrm{~mm}$ or more, it 
Flap Selection Algorithm Based on BMI Watanabe et al.

Table 1 Demographics of female-to-male transgenders according to flap types

\begin{tabular}{|c|c|c|c|c|}
\hline \multirow[t]{2}{*}{ Characteristics } & \multicolumn{3}{|c|}{ Flaps for phalloplasty } & \multirow[t]{2}{*}{$p$} \\
\hline & RF group $(n=10)$ & ALT group $(n=8)$ & FC group $(n=15)$ & \\
\hline $\begin{array}{l}\text { Age (y) } \\
\text { Mean (SD) }\end{array}$ & $32.2(7.9)$ & $34.7(6.6)$ & $32.2(5.1)$ & $>0.05$ \\
\hline $\begin{array}{l}\text { Body mass index }\left(\mathrm{kg} / \mathrm{m}^{2}\right) \\
\text { Mean (SD) }\end{array}$ & $25.9(3.3)$ & $21.7(0.9)$ & $22.2(4.5)$ & $<0.05^{a}$ \\
\hline $\begin{array}{l}\text { Follow-up period (mo) } \\
\text { Median (range) }\end{array}$ & $66(12-144)$ & $24(12-24)$ & $54(12-162)$ & \\
\hline \multicolumn{5}{|l|}{$\begin{array}{l}\text { Early complication } \\
n(\%)\end{array}$} \\
\hline Thrombosis & $0(0)$ & $0(0)$ & $4(26)$ & $>0.05$ \\
\hline Flap necrosis & $0(0)$ & $0(0)$ & $1(6)$ & $>0.05$ \\
\hline \multicolumn{5}{|l|}{$\begin{array}{l}\text { Late complication } \\
n(\%)\end{array}$} \\
\hline Fistula & $8(80)$ & $4(50)$ & $5(33)$ & $>0.05$ \\
\hline Calculus & $0(0)$ & $0(0)$ & $2(13)$ & $>0.05$ \\
\hline \multicolumn{5}{|l|}{$\begin{array}{l}\text { Re-operations } \\
n(\%)\end{array}$} \\
\hline Fistulectomy & $8(80)$ & $4(50)$ & $5(33)$ & $>0.05$ \\
\hline Open resection depilation & $0(0)$ & $0(0)$ & $2(13)$ & $>0.05$ \\
\hline \multicolumn{5}{|l|}{$\begin{array}{l}\text { Esthetic acceptability } \\
\text { Mean (SD) }\end{array}$} \\
\hline${ }^{\mathrm{b}}$ Evaluation scores $(1-4)$ & $3.7(0.4)$ & $3.5(0.9)$ & $2.5(1.2)$ & $<0.05^{a}$ \\
\hline $\begin{array}{l}\text { Tactile or erogenous sensibility } \\
n(\%)\end{array}$ & $10(100)$ & $8(100)$ & $6(40)$ & $<0.05^{a}$ \\
\hline $\begin{array}{l}\text { Standing urination } \\
n(\%)\end{array}$ & $8(80)$ & $6(75)$ & $13(87)$ & $>0.05$ \\
\hline
\end{tabular}

Abbreviations: ALT, anterolateral thigh flap; FC, flap combination; RF, radial forearm flap; SD, standard deviation. ${ }^{\mathrm{a}} p<0.05$ (Kruskal-Wallis test).

bEvaluation scores (1: strongly disagree, 2: disagree, 3: agree, 4: strongly agree).

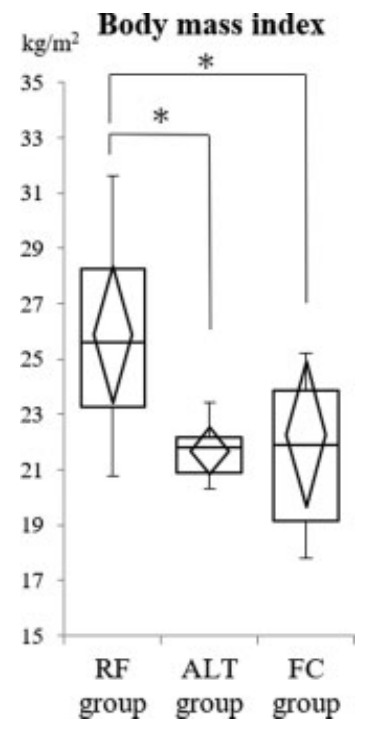

Fig. 2 The RF group has a higher BMI value than the other two groups. Significant differences are found between the RF and FC groups and between the RF and ALT groups. ( ${ }^{*} p<0.05$, Mann-Whitney U-test). ALT, anterolateral thigh; BMI, body mass index; FC, flap combination; $\mathrm{RF}$, radial forearm.

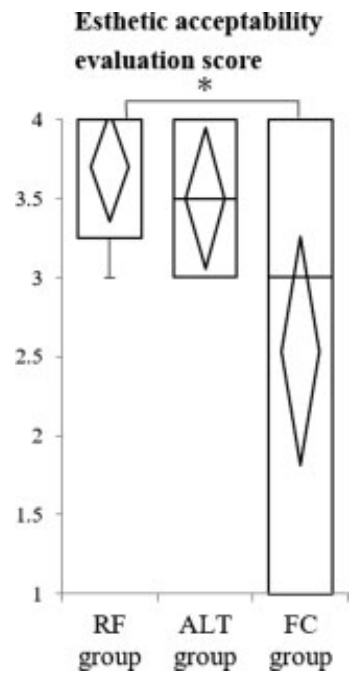

Fig. 3 The FC group has the lowest score among the three groups, and a significant difference is observed between the RF and FC groups. $\left({ }^{*} p<0.05\right.$, Mann-Whitney U-test). ALT, anterolateral thigh; FC, flap combination; RF, radial forearm. 
does not fit within the angiosome territory and is more likely to cause necrosis at the flap margin. In that case, we use the FC phalloplasty. Therefore, we believe that the thickness of the flap should be within $7 \mathrm{~mm}$. If the flap is less than $3 \mathrm{~mm}$ thick, the penis will be too thin which is a cosmetic problem. Therefore, in the case of flap thickness more than $7 \mathrm{~mm}$, additional procedures for flap thinning may be required for phalloplasty. However, this procedure may cause impaired blood flow, interfere with tactile or erogenous sensibility, and make penile morphogenesis difficult. ${ }^{18,19}$

Our results suggest that BMI effected flap choices for phalloplasty because of the correlation relation between BMI and skin thickness. ${ }^{22,23}$ In our series, the BMI of patients using ALT flaps or FC was significantly lower than that of patients using RF flaps. In the case of high BMI, the RF flap was the first choice because in our all cases, forearm skin thickness was less than $7 \mathrm{~mm}$ despite high BMI. On the contrary, when the BMI was low, the flap thickness of the RF flap was too low ( $<3 \mathrm{~mm}$ ) for an ideal phalloplasty. For these cases, ALT flap or FC was selected in terms of the relation between BMI and flap thickness. FC phalloplasty is combined several flaps, so it is possible to harvest flaps considering body skin thickness. In our series, FC becomes an option between ALT flap and RF flap in terms of flap choice based on BMI.

RF flap feasibility requires the Allen test to confirm that the radial artery can be safely harvested. If the Allen test is negative, RF flaps cannot be used. ${ }^{19}$ For these cases, opposite RF or ALT flap should be selected. An ALT flap can be used with a pedicled flap and has the advantage of not requiring microsurgery. ${ }^{14,17}$ The ALT flap differs in vascular anatomy, and it is important to confirm the existence and location of perforator vessels using color Doppler ultrasonography and MDCTA preoperatively. ${ }^{17}$ However, if the perforator vessel diameter is small, only one or no perforating branch exists, and if the vascular pedicle length is short, the feasibility of a pedicled ALT flap is low. In these cases, the opposite ALT flap or FC should be selected.

Our results indicated the lowest scores of esthetic acceptability evaluation scores in the FC group, and no significant difference was observed in the esthetic acceptability evaluation scores between the RF and ALT groups. FC is a technique of combining various flaps; however, the technique is more complicated than the "tube-within-a-tube" flap design phalloplasty. ${ }^{15}$ In the FC group, flaps harvested from the skin of various thicknesses, such as the lower abdomen, thighs, and forearms, are combined. Therefore, it becomes challenging to adjust the shape because it depends on the choice of flaps to be combined, and the reconstructed penis may become too large or too small.

However, in standard deviation comparison of esthetic acceptability evaluation scores, the FC group (1.2) was higher than the RF (0.4) and ALT group (0.9), and in some cases of FC, esthetic acceptability evaluation scores were as high as RF and ALT ( - Fig. 3). In high-score FC cases, the urethral surface was constructed with a thin flap, such as an RF flap or SCIP flap, and the foreskin was reconstructed with a thick flap, such as an ALT flap or DIEP flap. In addition, FC cases that required thinning of flaps had lower scores. This suggests that it was difficult to perform phalloplasty by thinning the flaps and adjusting the thickness during surgery. Therefore, considering the required flap thickness is from 4 to $6 \mathrm{~mm}$, the combined flap thickness that affects the shape of the reconstructed phallus should be considered.

Perceptual recovery has been reported to be good and consistent with both the RF and ALT flaps. ${ }^{7,14,17-19}$ In our series, the RF and ALT groups reported good tactile or erogenous sensibility. However, in the FC group, six patients did not achieve tactile or erogenous sensibility after the procedure. In FC phalloplasty, when a DIEP flap and an SCIP flap are used, including the sensory nerve in the flap, it becomes challenging. One of the solutions to this shortcoming is to leave the clitoris at the base of the reconstructed penis or to combine the innervated ALT flap or RF flap. ${ }^{15}$

Understanding the scarring and dysfunction of the donor site when choosing the appropriate flap will benefit the patient. Phalloplasty with a single flap and the "tube-within-a-tube" reconstruction design may cause serious complications when large scars are left at the donor site. ${ }^{8,18,19}$ In FC phalloplasty, various flaps are taken from multiple donor sites, thereby reducing the scarring at each donor site. ${ }^{15}$ The patient's choice of donor sites may rely on reducing donor site morbidity, and choosing different donor sites can be beneficial to the patient.

Based on our results, the factors affecting preoperative flap selection were BMI, the confirmation of perforator vessel location included in the flaps, and tactile or erogenous sensibility. Considering these results, an alternative algorithm for phalloplasty for flap selection is suggested in -Fig. 4. When the BMI is low, especially when the thickness of the lateral aspect of the thigh is less than $6 \mathrm{~mm}$, the ALT flap is selected after identifying the perforator vessels on the MDCTA image. When the perforator vessels of the ALT flap cannot be identified on the MDCTA image, the RF flap is selected after confirming the positive Allen test. FC is selected if the patient refuses postoperative scarring of the thigh or when the thickness of the forearm area is less than

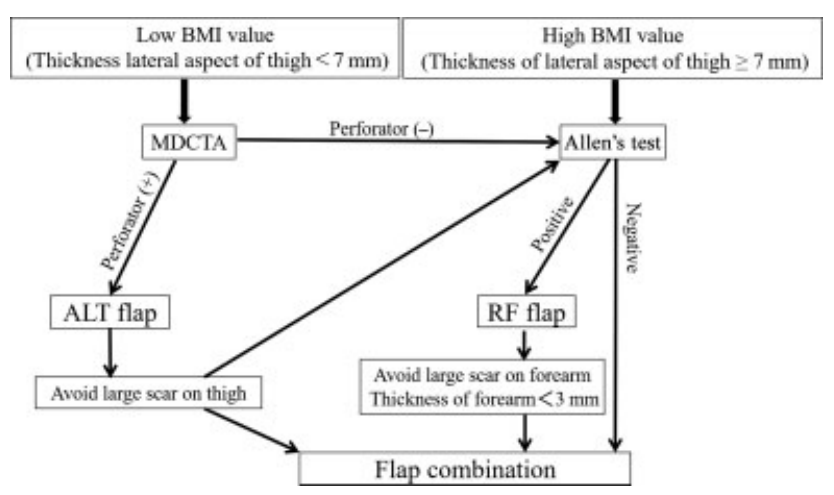

Fig. 4 Algorithm for selecting a flap for phalloplasty in female-tomale transgender. Perforator (+); two or more perforator vessels within the lateral thigh area can be identified on MDCTA images. Perforator (-); one or no perforator vessel within the lateral thigh area can be identified on MDCTA images. ALT, anterolateral thigh; BMI, body mass index; MDCTA, multidetector computed tomographic angiography; RF, radial forearm. 
$3 \mathrm{~mm}$. When the BMI is high, especially when the thickness of the lateral aspect of the thigh is more than $7 \mathrm{~mm}$, the RF flap is selected after confirming the positive Allen test. When the Allen test is negative or if the patient rejects postoperative scarring of the forearm area, FC should be selected.

\section{Limitations}

Limitations of this study include the relatively small number of cases which were selected from the same facility: the inclusion of only three types of flaps (RF flap, ALT flap, and FC) and the lack of patient satisfaction data and functional assessment. No established procedure was conducted to assess the perception of patients regarding the reconstructed penis and evaluate patient satisfaction at the facility.

\section{Conclusion}

We proposed an algorithm for flap selection in phalloplasty. FC becomes an option between ALT flap and RF flap in terms of flap choice based on BMI. FC was the second choice in terms of esthetic acceptability, tactile, or sensuality, but when choosing flaps considering skin thickness and sensory recovery, FC becomes an ideal phalloplasty as high score as phalloplasty using ALT or RF flaps. If reasons for a specific flap selection are clearer, patients and surgeons will gain better understanding of the procedures and the consequent benefits to the patient.

\section{Conflict of Interest}

None declared.

\section{References}

1 Bogoras N. Uber die volle plastische Wiederherstellung eines zum koitus fahigen penis. Zentralbl Chir 1936;22:1271-1276

2 Maltz M. Maltz Reparative Technique for the Penis. New York, NY: Froben Press; 1946:278-279

3 Gillies H, Harrison RJ. Congenital absence of the penis. Br J Plast Surg 1948;1(01):8-28

4 Chappell BS. Utilization of scrotum in reconstruction of penis. J Urol 1953;69(05):703-707

5 Kaplan I, Wesser D. A rapid method for constructing a functional sensitive penis. Br J Plast Surg 1971;24(04):342-344

6 Orticochea M. A new method of total reconstruction of the penis. Br J Plast Surg 1972;25(04):347-366
7 Chang TS, Hwang WY. Forearm flap in one-stage reconstruction of the penis. Plast Reconstr Surg 1984;74(02):251-258

8 Gilbert DA, Horton CE, Terzis JK, Devine CJ Jr, Winslow BH, Devine PC. New concepts in phallic reconstruction. Ann Plast Surg 1987; 18(02):128-136

9 Harashina T, Inoue T, Tanaka I, Imai K, Hatoko M. Reconstruction of penis with free deltoid flap. Br J Plast Surg 1990;43(02): 217-222

10 Semple JL, Boyd JB, Farrow GA, Robinette MA. The "cricket bat" flap: a one-stage free forearm flap phalloplasty. Plast Reconstr Surg 1991;88(03):514-519

11 Upton J, Mutimer KL, Loughlin K, Ritchie J. Penile reconstruction using the lateral arm flap. J R Coll Surg Edinb 1987;32(02):97-101

12 Sadove RC, Sengezer M, McRoberts JW, Wells MD. One-stage total penile reconstruction with a free sensate osteocutaneous fibula flap. Plast Reconstr Surg 1993;92(07):1314-1323, discussion 1324-1325

13 Lai C-S, Chou C-K, Yang CC, Lin SD. Immediate reconstruction of the penis with an iliac flap. Br J Plast Surg 1990;43(05):621-624

14 Felici N, Felici A. A new phalloplasty technique: the free anterolateral thigh flap phalloplasty. J Plast Reconstr Aesthet Surg 2006; 59(02):153-157

15 Namba Y, Watanabe T, Kimata Y. Flap combination phalloplasty in female-to-male transsexuals. J Sex Med 2019;16(06):934-941

16 Monstrey S, Hoebeke P, Selvaggi G, et al. Penile reconstruction: is the radial forearm flap really the standard technique? Plast Reconstr Surg 2009;124(02):510-518

17 Hasegawa K, Namba Y, Kimata Y. Phalloplasty with an innervated island pedicled anterolateral thigh flap in a female-to-male transsexual. Acta Med Okayama 2013;67(05):325-331

18 Heston AL, Esmonde NO, Dugi DD III, Berli JU. Phalloplasty: techniques and outcomes. Transl Androl Urol 2019;8(03):254-265

19 Morrison SD, Shakir A, Vyas KS, Kirby J, Crane CN, Lee GK. Phalloplasty: a review of techniques and outcomes. Plast Reconstr Surg 2016;138(03):594-615

20 Matsumoto Y, Abe T, Ikeda K, et al; The Japanese Society of Psychiatry and Neurology The diagnostic and therapeutic guidelines for patients with gender identity disorder [in Japanese]. Seishin Shinkeigaku Zasshi 2012;114(11):1250-1266

21 Frey JD, Poudrier G, Chiodo MV, Hazen A. A systematic review of metoidioplasty and radial forearm flap phalloplasty in female-tomale transgender genital reconstruction: is the "ideal" neophallus an achievable goal? Plast Reconstr Surg Glob Open 2016;4 (12):e1131

22 Murakami M, Hikima R, Arai S, Yamazaki K, Iizuka S, Tochihara Y. Short-term longitudinal changes in subcutaneous fat distribution and body size among Japanese women in the third decade of life. Appl Human Sci 1999;18(04):141-149

23 Saito H, Tamura T. Fat distribution in Japanese women. Part 2. Fat thickness of extremities and classification of fat distribution of whole body [in Japanese]. Ann Physiol Anthropol 1994;13(04): 183-196 